\title{
Transcaval access for transcatheter aortic valve replacement: Use of Brockenbrough needle
}

Abstract: The impossibility of obtaining an access to the implant of the percutaneous aortic valve is a great challenge. The transcaval access is an alternative option that is used less frequently in patients with a high risk of surgical access. The usual transcaval access is described with the use of a rigid tip wire, aided by microcatheter and electrosurgery to across from cava vein to abdominal aorta. This clinical case shows a technical alternative to the transcaval access, with the help of Brockenbrough needle, commonly used to perform the transseptal puncture.

Keywords: Transcatheter aortfic valve Brockenbrough needle - Transcaval access $=$ Anesthesia

Introduction: Transcatheter aortic valve implantation reduces the morbidity and mortality of surgical aortic valve replacement in high and intermediate risk patients [1,2]. Even, the current evidence shows benefits in mortality, stroke and rehospitalizations in low risk patients in comparison to surgery in the short term [3]. The transcaval approach to implantation of the transcatheter valve is a challenge in patients that have an inadequate size of the femoral arteries and a high risk of complications in surgical access, such as transapical, aortic or subclavian [4].
Christian Dauvergne, Pablo Antileo*, Jorge Sandoval

Instituto Nacional del Tórax, Catheterization Laboratory, 171 José Manuel Infante Street, 2nd Floor, Providencia, Santiago, Chile

*Author for correspondence: Email: pablo.antileo@gmail.com Received date: December 26, 2019 Accepted date: January 09, 2020 Publication date: January 17,2020

\section{Case Presentation}

$\mathrm{He}$ is an eighty-three-years-old man; very fragile, with a history of hypertension, chronic obstructive lung disease, chronic renal insufficiency, bicameral pacemaker and atrial fib rillation. The current clinical status is congestive Heart Failure NYHA functional class III-IV. Echocardiography showed severe aortic stenosis, low flow and low gradient with $20 \%$ ejection fraction. Aortic valve area was $0.85 \mathrm{~cm}^{2}$. Stress Echocardiography up to $20 \mathrm{ug} / \mathrm{Kg} / \mathrm{min}$ of dobutamine showed a $4.2 \mathrm{~m} / \mathrm{s}$ velocity and $42 \mathrm{mmHg}$ medium gradient. Due to a high surgical risk (STS score 10\%), the heart team decided to perform a percutaneous aortic valve implantation procedure. Vascular access assessment with computed tomography (CT) showed minimum diameter of $5 \mathrm{~mm}$ in left femoral iliac artery. The CT showed an aortic annulus of $6.7 \mathrm{~cm}^{2}$, low calcium and good heights of coronaries. The $\mathrm{p}$ rocedure $\mathrm{w}$ as $\mathrm{p}$ lanned with this approach: under general anesthesia, with support of fluoroscopy and image fusion, and we used the left artery access as first try. It was not possible to advance with 20 $F$ dilator for femoral access, due to circumferential calcificationi $\mathrm{n}$ the left iliac artery. The patient had t ortuosity o f $s$ ubclavian a rtery a nd the surgeon was not an expert in aortic access, and there was a contraindication for transapical access due to pulmonary disease. So we decided to convert to transcaval access. Throught the right femoral venous access, a Fast-Cath $^{\oplus}$ SL2 sheath (St. Jude Medical) and a Brockenbrough needle were advanced (Figure 1).

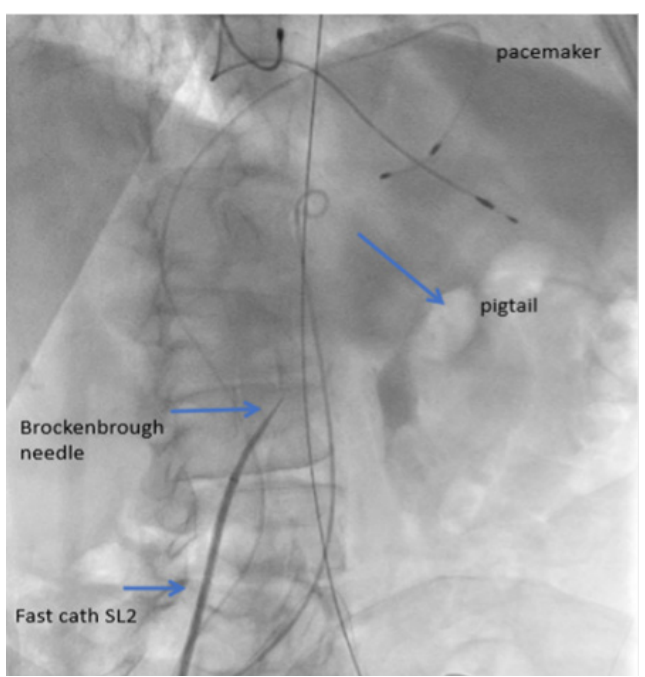

Figure 1: Brockenbrough needle is located in cava vein to cross to abdominal aorta.

We used the "pigtail" catheter as reference in abdominal aorta through right femoral access. The pacemaker was located in right ventricle through right femoral vein. The choice of the crossing point was performed with angiography (image fusion with the CT using the Philips VesselNavigator ${ }^{\otimes}$ software) and confirmed with the pressure in the tips of the needle (Figure 2). 


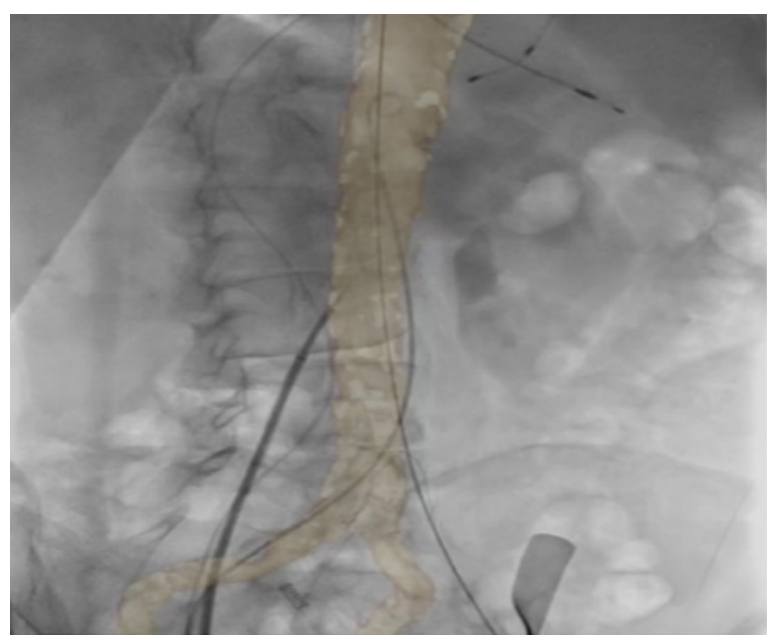

Figure 2: Imagen fusion between angiography and computed tomography.

After, a whisper ${ }^{\circ}$ wire (Abbot Vascular) was advanced (Video 1) and exchanged for a stiffer support wire (Lunderquist ${ }^{\circ}$, Cook Medical) towards to thoracic aorta, and then the FastCath $^{\circ}$ SL2 with the needle into it were moved through the abdominal and thoracic aorta. We advanced the delivery system (Video 2) and located the Edwards Sapien $\mathrm{XT}^{\circ} 29 \mathrm{~mm}$. The valve was directly implanted without complications, the transaortic gradient was 4 $\mathrm{mmHg}$ without aortic regurgitation (Video 3). The transcaval access was closed with a $10 \mathrm{~mm}$ Amplatzer muscular VSD occluder ${ }^{\circ}$ The device deployment was performed from the artery to the vein. The position of the delivery device was confirmed. The final sustraction angiography control did not show flow from aorta to cava vein (Video 4). The patient had a good clinical evolution after the procedure and without access complications.

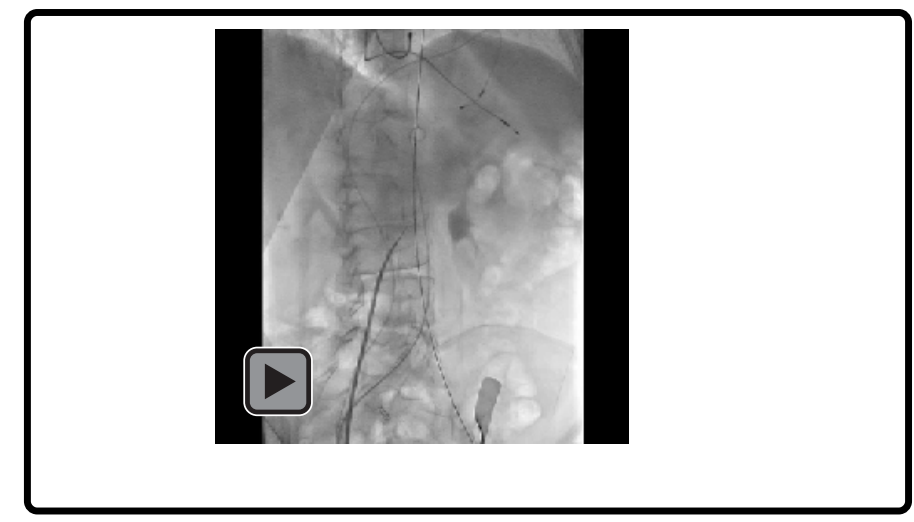

Video: 1

Video: 3

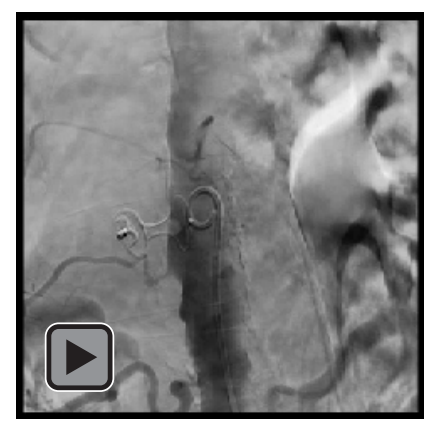

Video: 4

\section{Discussion}

Transcaval access is a completely percutaneous route from the inferior cava vein, through the retroperitoneal interstitial space and 


\section{Case Report}

into the abdominal aorta to allow therapeutic procedures, such as transcatheter aortic valve replacement. It is an alternative to access to the femoral artery when the iliofemoral arteries are too small or too diseased to accommodate the desired introducer sheath. Transcaval access to the aorta is based on the observation that the interstitial hydrostatic pressure exceeds the venous pressure. As a result, in the retroperitoneal space, blood that leaves the abdominal aortic returns to the venous circulation through a hole in the cava vein, instead of being accumulated as hemorrhage. This physiology allows the large transcave sheaths to be temporarily removed during tract repair and allows the aortocaval tracts to be closed with nitinol occluding devices [5]. The traditional transcaval access is described with the use of a rigid-tip coronary CTO guidewire, aided by microcatheter and electrocautery, which is advanced in a calcium-free target in the aorta. Then, the system is exchanged by stiff support guide and delivery system is advanced through a fistula cava-aorta to deploy the valve [6]. In our case, we decided to use the Brockenbrough needle. This allowed us, with the help of pressure measurement in the tip of the needle and the image fusion, not only to know when we entered the abdominal aorta, but also having a better support. It was very important to choose a zone without calcium and avoiding iliac bifurcation and renal arteries to proximal. The next steps were the usual, and finally, the closure of the fistula aortic vein was made and there was not access complications. In the literature, there is no reference to transcaval access with the use of Brockenbrought needle.

\section{Conclusion}

We concluded that CT information has a paramount importance for planning the procedure, and the image fusion with the angiography was useful to be oriented about the transcaval access. The Brockenbrough needle is secure and accurate for crossing the aorta, gives instantaneous pressure measurement, and it is a novel alternative to the traditional method for crossing the transcaval access.

\section{Conflicts of Interest}

The authors declare that they have no conflict of interest.

\section{References}

1. Leon MB, Smith CR, Mack MJ, et al. Transcatheter aortic-valve implantation for aortic stenosis in patients who cannot undergo surgery. N Engl J Med. 2010;363:1597-1607

2. Leon MB, Smith CR, Mack MJ, et al. Transcatheter or Surgical Aortic-Valve Replacement in Intermediate-Risk Patients. N Engl J Med. 2016;374:1609-1620.

3. Mack MJ, Leon MB, Thourani VH, et al. Replacement with a BalloonExpandable Valve in Low-Risk Patients. N Eng J Med. 2019;380:1695-1705

4. Lederman RJ, O’Neill WW, Greenbaum AB, et al. Transcaval Access for TAVR Across a Polyester Aortic Graft. Catheter Cardiovasc Interv. 2015;85:1270 1273.

5. Lederman RJ, Babaliaros VC, Rogers T, et al. The fate of transcaval access tracts: 12- Month Results of the Prospective NHLBI Transcaval Transcatheter Aortic Valve Replacement Study. JACC Cardiovasc Interv. 2019;12:448-456.

6. Lederman RJ, Babaliaros VC, Greenbaum AB et al. How to Perform Transcaval Access and Closure for Transcatheter Aortic Valve Implantation. Catheter Cardiovasc Interv. 2015;86: 1242-1254. 